\title{
Local Method for Curved Edges and Corners.
}

\author{
R T Shann \& J P Oakley \\ Depts Medical Illustration \& Electrical Engineering \\ University of Manchester \\ Manchester M13 9WL
}

\begin{abstract}
A new method of detecting features in Gaussian smoothed images is described. Applied to the simple case of marking the Canny edge pixels the method gives an improved response at corners where traditional methods have problems. Moreover, the method permits marking of more sophisticated features at essentially no extra cost. Additional information available includes local curvature estimates for each edge, direct (local) identification of occluded edges and marking and characterising comers on edges. The performance of the method on real images is compared with the PlesseyHarris comer detector. We find that the quality and sensitivity of these corner detection methods are similar, with the new method giving information on the orientation and opening angle of the corners. Finally it is shown how ellipse detection in noisy images is made possible using this curvature information.
\end{abstract}

\section{Introduction}

The Canny edge detector [1] consists in practice of the following:

(a) Gaussian convolutions to yield $\mathrm{x}$ and $\mathrm{y}$ gradients of the Gaussian smoothed image (GSI)

(b) Checking adjacent pixels in the GSI for maxima of the gradient magnitude in the (discretized) gradient direction. This step is a crude substitution for computing further derivatives, which is where our new method makes an impovement.

At least two significantly different implementations of this scheme are in use. In one (eg the one originating at AIVRU Sheffield) only pixels above and to the side are used to perform step (b) above. In the other (eg from RAL Didcot) the diagonal pixels are used as well. Because these are discrete methods they have problems when the direction of the edge is changing quickly. The first approach leaves gaps at comers while the second gives spurious short edges. These problems at corners are not serious in a typical application because non-local processing will generally be applied to break up edges into segments at comers. However if one is hoping to extract a more detailed local characterisation of the image behaviour than just edge position and orientation then we need a more sophisticated treatment of the higher derivatives.

New Computational Scheme

The new method uses a continuous rather than a discrete image (as introduced in [3], [4]). We take as our Gaussian smoothed image the analytic function

$$
\tilde{l}(x, y)=\sum_{i} I_{j} j G(x-i, y-j)
$$


Here $(x, y)$ are floating point coordinates in the image plane, lij is the $i j$ th pixel greylevel and $G$ is the Gaussian filter kernel. In terms of this function steps (a) \& (b) of the Canny scheme above can be replaced by answering the following question for each pixel $(i, j)$

'Does $\tilde{I}(x, y)$ have a maximum in the gradient direction of its gradient magnitude in the region bounded by $x=i+1-0.5, y=j+1-0.5$ ?'

The conditions to be satisfied for (2) to be true have appeared in the literature [5],[6], in terms of higher spatial derivatives of $\tilde{I}$. Since we can compute the derivatives using

$$
\tilde{I}_{x}=\sum_{i j} I_{i j} G_{x}(x-i, y-j) \quad \text { etc }
$$

we have in principle a method of answering (2) and thereby replacing steps (a)\&(b) of the traditional schemes. The method we have adopted is as follows: First we compute (3) at the pixels centres for as many derivatives as we need. Then for each pixel

(a) Approximate the condition for (2) by a quadratic form in $\mathrm{x}$ and $\mathrm{y}$ using Taylor's expansion.

(b) The condition is now a conic section, so we can check analytically whether it crosses the given pixel.

In this implementation the computations of (3) were done using a simple FFT algorithm. At sharp comers the traditional methods either leave gaps or add short stubby edges while the new method gives the correct response.

\section{Computing Corners}

As mentioned earlier one could not normally justify using this method for its better treatment of high curvature edges. However, once the work of computing (3) has been done we have created a mine of local information about the image surface, and as an example we will show here how to use the information to detect corners on edges. We can compute the radius of curvature $\mathrm{R}$ using (see [8])

$$
\mathrm{R}^{-1}=\nabla \cdot \frac{\nabla \tilde{I}}{|\nabla \tilde{I}|}
$$

Now what characterises a comer (as opposed to any point on an arc) is that the curvature goes through a maximum or minimum in the direction along the edge. By the usual argument this occurs at a zero of the second derivative taken in the direction along the edge, and as before we use Taylor's theorem to enable us to compute an 


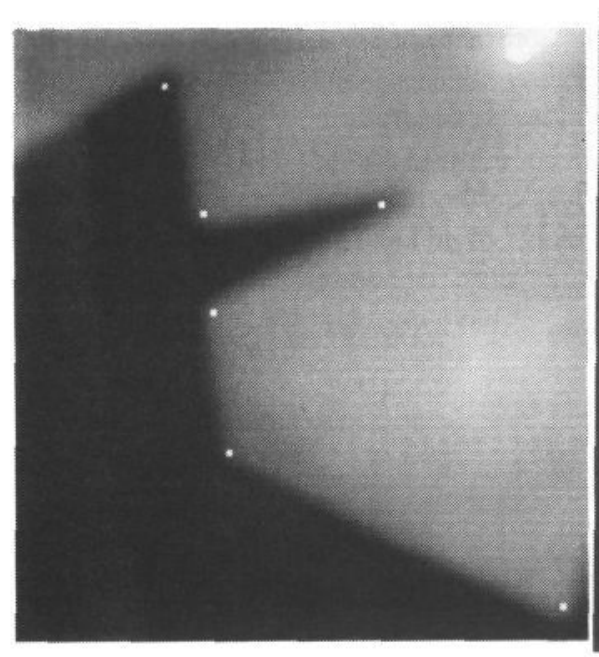

Fig 1 Image with simple geometrical features.

The bright spots mark the corners detected by the $\sigma=2$ Plessey-Harris corner detector

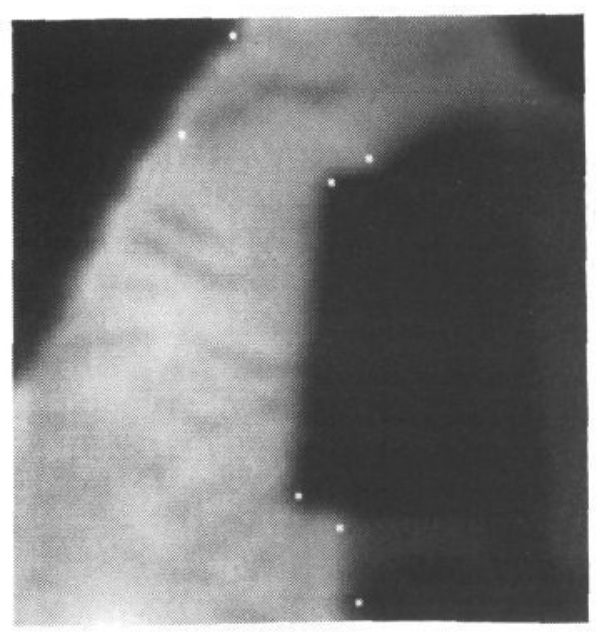

Fig 3 Image of marker pen against textured background. The bright spots mark the corners detected by the $\sigma=2$ Plessey-Harris corner detector

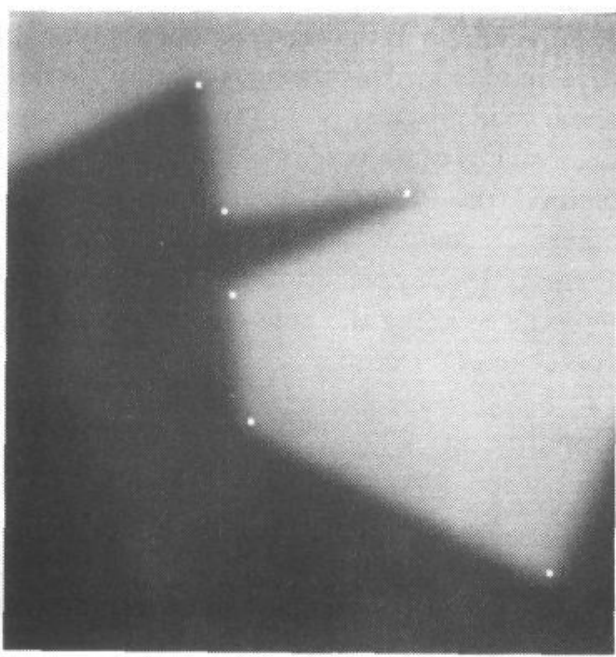

Fig 2 Image as Fig 1 The bright spots mark the corners detected by the method of this paper using the same $\sigma=2$ pixels

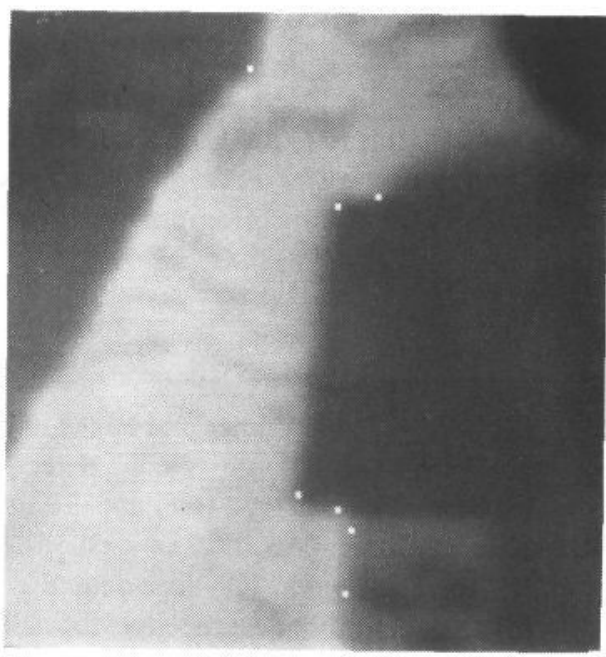

Fig 4 Image as Fig 3 The bright spots mark the corners detected by the method of this paper using the same $\sigma=2$ pixels 
approximation to the zero contour analytically. A corner is then declared wherever this zero contour crosses the zero contour (ie the Canny edge) already computed, provided always the crossing point is within the pixel under consideration. Figs (1) - (4) show the result of marking corners both with the current method and a well-known standard method, the Plessey-Harris comer detector [7] [8], both with the same sigma of 2 pixels. The output is quite similar. In the first image there are four colinear comers and a sharp 19 degree corner. These geometrical properties are consistently better reproduced using the new method. In addition we have an estimate for the centre of curvature of the comer.

\section{Conclusion}

The same basic approach can be used to determine points where the local surface has any particular characteristic shape. For example, when an edge is occluded the zero contour of the third directional derivative of the gradient magnitude crosses that of the second. Applied to the marker pen image in fig 3 this criterion detects the junction of the barrel and cap as the strongest occlusion. Low gradient points (eg ridges, spots) can be approached by the same methods. Finally, the curvature information has been successfully used to detect circles, and (using pairwise search) ellipses in noisy images (where the edges are broken by the noise) using the local curvature information to vote for circle/ellipse centres.

\section{Acknowledgements}

This work has been done under funds provided by Central Manchester Health Authority. Thanks to Chris Harris for help in implementing his comer detector, and to Maria Petrou of the University of Surrey and to the Oxford Robotics group for copies of conventional Canny programs, and to Stephen Pollard for discussions on his algorithm.

\section{References}

[1] Canny 'Finding lines and edges in Images' MIT Tech report 720 (1983)

[2] Petrou 'On the optimal edge detector', Proceedings AVC88 191-196 (1988)

[3] Shann \& Oakley "Novel approach to boundary finding" IVC

[4] Oakley \& Shann "An efficient method ..." IVC (to appear October 1991)

[5] Torre \& Poggio "On edge detection" IEEE TPAMI 2,147 (1986)

[6] Clark "Authenticating edges ..." IEEE TPAMI 11,43 (1989)

[7] Harris 'Combined comer and edge detectior' Proceedings of AVC88 p 147152

[8] Noble 'Finding Comers' IVC 6(2) 121-128 (1988) 